\section{PWE-020 USING MICROARRAY DATA TO ELUCIDATE THE MOLECULAR PHENOTYPE OF A NOVEL ONCOGENE AND IDENTIFY A THERAPEUTIC STRATEGY}

doi:10.1136/gutjnl-2012-302514d.20

${ }^{1} \mathrm{C}-\mathrm{A} J \mathrm{Ong},{ }^{2} \mathrm{~N}$ B Shannon, ${ }^{*}{ }^{2} \mathrm{O} \mathrm{M}$ Rueda, ${ }^{3} \mathrm{R}$ Hardwick, ${ }^{2} \mathrm{C}$ Caldas, ${ }^{1} \mathrm{R} \mathrm{C}$ Fitzgerald. ${ }^{1}$ MRC Cancer Cell Unit, Cambridge, UK; ${ }^{2}$ Cambridge Research Institute, Cambridge, UK; ${ }^{3}$ Cambridge Oesophago-gastric Centre, Cambridge, UK

Introduction The incidence of esophageal and junctional adenocarcinoma has increased sixfold in the past 30 years. Despite aggressive chemotherapy and attempts at curative surgery, the 5 year survival rate remains at $20 \%$. Unlike other epithelial cancers, targeted therapy is limited. We have identified TRIM44 as a putatative oncogene that is amplified in $8 \%$ of EA and $6 \%$ of breast cancers.

Methods The aims of this project were to elucidate the molecular phenotype underlying the oncogenic role of TRIM44 as well as suggest ways to therapeutically target TRIM44 dysregulation.

Results Using three microarray datasets representing EA $(n=37$, $n=64)$ and breast ( $n=997$ ) we performed gene set enrichment analysis to identify signalling pathways dysregulated with TRIM44 high expression (EA and breast) or amplification (breast only). High expression of TRIM44 was associated with over-enrichment of targets of the mTOR pathway consistent across all three data sets. This association was validated using expression microarrays in a cell line (HSC39) with amplification of TRIM44 treated with siRNA. Using phosphorylation of p70S6K as a readout of mTOR activity we validated the link between TRIM44 and the MTOR pathway; knockdown of TRIM44 using siRNA in HSC39 and a cell line with high expression of TRIM44 (JIMT-1) resulted in a decrease in pathway activity. The connectivity map (http://www.broadinstitute.org/cmap/), a collection of expression array data derived from lines treated with bioactive small molecules was queried using signatures generated from the microarray data representing genes positively or negatively associated with TRIM44 (breast, EA, HSC39 +SiRNA datasets). The top consistent hits were sirolimus, an mTOR inhibitor and analogue of rapamycin and LY-294002, a PI3K inhibitor. These hits represent small molecules predicted to reverse the effects of high TRIM44 expression. Treatment with inhibitors in HSC39 and JIMT-1 demonstrated that these lines were highly sensitive (IC50<30 nM) to rapamycin, but less sensitive to PI3K inhibition (IC50>400 nM), consistent with a link at the level of mTOR

Conclusion We have demonstrated the ability to identify a previously unknown association between TRIM44 and the mTOR pathway using expression and copy number data. This phenotype was validated in cell line experiments and highlighted a potential therapeutic strategy using analogues of rapamycin, a small molecule inhibitor of the mTOR pathway, which are currently in clinical use as immunosuppressants and in clinical trials for other cancer types.

Competing interests None declared.

\section{PWE-021 SECONDARY CARE COSTS IN OESOPHAGOGASTRIC CANCER IN THE UK: A SINGLE CENTRE MICRO-COSTING STUDY}

doi:10.1136/gutjnl-2012-302514d.21

${ }^{1} \mathrm{~N}$ Stern, ${ }^{*}{ }^{2} \mathrm{~A}$ Bassi, ${ }^{1,3} \mathrm{~K}$ Bodger. ${ }^{1}$ Department of Gastroenterology, Aintree University Hospital NHS Foundation Trust, Liverpool, UK; ${ }^{2}$ Department of Gastroenterology, St Helens and Knowsley Hospitals NHS Trust, Prescot, UK; ${ }^{3}$ Institute of Translational Medicine, University of Liverpool, Liverpool, UK

Introduction Published data describing the scale, profile and determinants of healthcare resource use for oesophago-gastric cancer
(OGC) are scarce, particularly for the UK. The National Cancer Plan (2000) encouraged greater use of imaging for staging and increased use of oncological therapies but the major modalities of palliation and curative surgery have changed little over the last decade. We have reported how emergency presentation predicts a poor outcome $^{1}$ and now aimed to study the effect of this mode of presentation on resource use and cost.

Methods Using cohorts of all patients presenting with OGC to a single university hospital trust (Aintree University Hospital, Liverpool, UK) over two, 2-year periods (July 1997-June 1999 and Jan 2001-Dec 2002) we audited all resource use from referral to diagnosis and 12 months post-diagnosis or death (whichever was sooner). Diagnostic and staging investigations, in-patient bed days and treatments including surgical, oncological and endoscopic were all itemised. Patient treatment was categorised according to initial intent. Individual hospital based unit costs or HRG tariffs (2010/ 2011 financial year) were applied to derive per-patient costs for the cohorts.

Results Of 333 patients identified, 320 had complete data to enable cost analysis. Median cost per-patient was £11965.94 with annualised costs to our institution of $£ 1.2$ million. Potentially curative treatment accounted for $32.5 \%$ of institutional costs, palliation $56.2 \%$ and supportive care $11.3 \%$. Per-patient costs [median (IOR)]: Curative treatment: $£ 11863.80$ (9701-17430); Palliative treatments: $£ 14288.52$ (10016-22710); Supportive care only: $£ 6619.96$ (3567-11778). Emergency presentations (31.2\% of cases) consumed $30.8 \%$ of cost despite shorter survival with significant increased costs per live day ( $£ 148.14$ vs $71.61, p<0.001)$, mostly due to longer inpatient stays. Linear regression of log transformed costs confirms that emergency presentation increased cost per live day independently of age or potentially curative treatment. Endoscopic interventions were used in 33.4\% patients.

Conclusion Managing OGC has a significant impact on healthcare resources with most expenditure is for those treated with palliative intent. Presenting as an emergency increases costs per live-day compared to elective presentation. These primary data can support modelling studies of cost-effectiveness of rival programmes or treatments for OGC.

Competing interests None declared.

\section{REFERENCE}

1. Shawihdi M, Stern N, Thompson E, et al. Emergency admission as a route for oesophago-gastric cancer diagnosis: a marker of poor outcome and a candidate quality indicator for local services. Gut 2011:60(Suppl 1):A30-1.

\section{PWE-022 COMPLETION RATES OF PALLIATIVE CHEMOTHERAPY ARE LOW IN PATIENTS WITH OESOPHAGO-GASTRIC CANCER: RESULTS FROM A NATIONAL AUDIT}

doi:10.1136/gutjnl-2012-302514d.22

${ }^{1} \mathrm{O}$ Groene, ${ }^{*} \mathrm{R}$ Hardwick, ${ }^{3} \mathrm{~S}$ Riley, ${ }^{4} \mathrm{~T}$ Crosby, ${ }^{5} \mathrm{~K}$ Greenaway, ${ }^{6} \mathrm{D}$ Cromwell. ${ }^{1}$ Health Services Research and Policy, London School of Hygiene \& Tropical Medicine, London, UK; ${ }^{2}$ Addenbrooke's, Cambridge, UK; ${ }^{3}$ Northern General Hospital, Sheffield, UK; ${ }^{4}$ Velindre Cancer Center, Cardiff, UK; ${ }^{5}$ NHS Information Centre, Leeds, UK; ${ }^{6}$ London School of Hygiene \& Tropical Medicine, London, UK

Introduction Palliative chemotherapy is routinely offered to patients diagnosed with locally advanced or metastatic oesophago-gastric cancer. Based on data from the National Oesophago-Gastric Cancer Audit (NOGCA), we describe the characteristics of patients being offered palliative chemotherapy, determine the proportion of patients completing treatment, and identify factors associated with treatment completion.

Methods The NOGCA prospectively collected data on patients diagnosed with invasive epithelial cancer of the oesophagus or 